\title{
Closed balls for interpolating quasi-polynomials
}

\author{
JIAJIN WEN ${ }^{1}$ and SUI SUN CHENG ${ }^{2}$ \\ ${ }^{1}$ College of Mathematics and Information Science, Chengdu University, \\ Chengdu 610106, P.R. China \\ ${ }^{2}$ Department of Mathematics, Tsing Hua University, Hsinchu, \\ Taiwan 30043, R.O. China \\ E-mails: wenjiajin623@163.com / sscheng@math.nthu.edu.tw
}

\begin{abstract}
The classic interpolation problem asks for polynomials to fit a set of given data. In this paper, quasi-polynomials are considered as interpolating functions passing through a set of spatial points. Existence and uniqueness is obtained by means of generalized Vandermonde determinants. By means of several estimates related to these determinants, we are also able to find closed balls for any given centers that enclose the approximating curves. By choosing proper centers based on the observed spatial points, these balls may lead us to applications such as satellite tracking and control.
\end{abstract}

Mathematical subject classification: 41A05.

Key words: interpolation, reference point, error bound, quasi-polynomial.

\section{Introduction}

Given a set of $m+1$ points $\left(x_{i}, y_{i}\right), i=0, \ldots, m$, where $x_{0}, x_{1}, \ldots, x_{m}$ are mutually distinct, the classical interpolation problem asks for a polynomial $p=p(x)$ of degree at most $m$ such that

$$
p\left(x_{i}\right)=y_{i}, \quad i=0,1,2, \ldots, m .
$$

The polynomial that does the job exists and is unique, and is called the Lagrange interpolating polynomial. Together with this existence and uniqueness theorem, 
there is now a fairly complete theory (see e.g. Davies [10]) associating the Vandermonde matrices, divided differences, error bounds, etc. with the classical interpolation problem.

In view of the many applications of the concept of interpolation, it is of interest to consider different types of interpolation functions. Among many others, in [5], "quasi-polynomials" as candidates of interpolating functions are considered. More specifically, let $\mathbf{R}$ and $\mathbf{C}$ be the set of real and complex numbers respectively. Let $\mathbf{b}_{1}, \mathbf{b}_{2}, \ldots, \mathbf{b}_{n} \in \mathbf{C}, \alpha_{1}, \alpha_{2}, \ldots, \alpha_{n} \in \mathbf{R}$ such that $0=\alpha_{1}<\alpha_{2}<\cdots<\alpha_{n}$. The function $\mathbf{f}:[0, \infty) \rightarrow \mathbf{C}$ defined by

$$
\mathbf{f}(u)=\mathbf{b}_{1} u^{\alpha_{1}}+\mathbf{b}_{2} u^{\alpha_{2}}+\cdots+\mathbf{b}_{n} u^{\alpha_{n}}, \quad u \in[0, \infty),
$$

is called a $\left(\alpha_{1}, \alpha_{2}, \ldots, \alpha_{n}\right)$-polynomial ${ }^{1}$. It is shown that given a set of data pairs $\left(x_{1}, \mathbf{y}_{1}\right),\left(x_{2}, \mathbf{y}_{2}\right), \ldots,\left(x_{n}, \mathbf{y}_{n}\right)$ where $0 \leq x_{1}<x_{2}<\cdots<x_{n}$ and $\mathbf{y}_{1}, \ldots$, $\mathbf{y}_{n} \in \mathbf{C}$, there then is a unique $\left(\alpha_{1}, \alpha_{2}, \ldots, \alpha_{n}\right)$-polynomial $\mathbf{f}$ that satisfies

$$
\mathbf{f}\left(x_{i}\right)=\mathbf{y}_{i}, \quad i=1,2, \ldots, n .
$$

Once existence is shown, it is then important to investigate the properties of the interpolating polynomial. Several properties are obtained in [5]. In particular, a bound for $|\mathbf{f}(u)|$, where $u \in\left[x_{1}, x_{n}\right]$, is obtained in [5].

In this paper, we will be interested in approximation of a spatial curve (described by a vector function) by $\left(\alpha_{1}, \alpha_{2}, \ldots, \alpha_{n}\right)$-polynomials, and their 'distances' from a reference point. More specifically, given a space curve in $\mathbf{R}^{m}$ described by a vector function $\mathbf{g}:\left[x_{1}, x_{n}\right] \rightarrow \mathbf{R}^{m}$. If $\mathbf{g}$ is unknown, but the set of data pairs $\left(x_{1}, \mathbf{y}_{1}\right),\left(x_{2}, \mathbf{y}_{2}\right), \ldots,\left(x_{n}, \mathbf{y}_{n}\right)$, where $0 \leq x_{1}<x_{2}<\cdots<x_{n}$ and $\mathbf{y}_{1}=\mathbf{g}\left(x_{1}\right), \ldots, \mathbf{y}_{n}=\mathbf{g}\left(x_{n}\right) \in \mathbf{R}^{m}$, are available, we are interested in the existence of a function $\mathbf{f}:[0, \infty) \rightarrow \mathbf{R}^{m}$ of the form

$$
\mathbf{f}(u)=\mathbf{d}_{1} u^{\alpha_{1}}+\mathbf{d}_{2} u^{\alpha_{2}}+\cdots+\mathbf{d}_{n} u^{\alpha_{n}}, u \in[0, \infty)
$$

where $\mathbf{d}_{1}, \mathbf{d}_{2}, \ldots, \mathbf{d}_{n} \in \mathbf{R}^{m}$ such that the condition

$$
\mathbf{f}\left(x_{i}\right)=\mathbf{y}_{i}, i=1,2, \ldots, n,
$$

\footnotetext{
${ }^{1}$ It is called a generalized polynomial in [5], but it is better to avoid this term since there are many generalizations of polynomials.
} 
is satisfied, as well as upper bounds for $\|\mathbf{f}(u)-\overline{\mathbf{y}}\|$, where $\|\cdot\|$ is the Euclidean norm for $\mathbf{R}^{m}$ and $\overline{\mathbf{y}}$ is a given vector in $\mathbf{R}^{m}$.

We plan to do the following. In the next section, we will take care of the existence and uniqueness of the desired function by introducing generalized Vandermonde determinants. Then we will state the main theorem of our paper. In Section 3, we will derive several preparatory results. Then in Section 4, our main theorem is proved. The final section is devoted to additional remarks and illustrative examples.

\section{Preliminary results}

To facilitate discussions, we recall several definitions and results. Throughout the rest of our discussions, we assume that $n \geq 2$ (to avoid trivial cases). Let $\mathbf{R}^{n}$ be the standard set of real $n$-vectors endowed with the usual linear structure and the Euclidean norm. An $n$-vector in $\mathbf{R}^{n}$ is indicated by $\mathbf{x}, \mathbf{a}, \mathbf{b}, \mathbf{c}, \alpha, \beta, \ldots$ etc. Given an $n$-vector, say $\mathbf{x}$, its components are indicated by $x_{1}, x_{2}, \ldots, x_{n}$, so that

$$
\mathbf{x}=\left(x_{1}, x_{2}, \ldots, x_{n}\right)^{\dagger},
$$

where the dagger indicates transposition. The difference vector $\Delta \mathbf{x}$ is defined by

$$
\Delta \mathbf{x}=\left((\Delta \mathbf{x})_{1},(\Delta \mathbf{x})_{2}, \ldots,(\Delta \mathbf{x})_{n-1}\right)^{\dagger}=\left(x_{2}-x_{1}, x_{3}-x_{2}, \ldots, x_{n}-x_{n-1}\right)^{\dagger} .
$$

For the sake of convenience, we also denote the $i$-th component $(\Delta \mathbf{x})_{i}$ of $\Delta \mathbf{x}$ by the forward difference $\Delta x_{i}$.

Several subsets of $\mathbf{R}^{n}$ will be used extensively. For this reason, we will set (cf. [1-9])

$$
\begin{gathered}
\mathbf{R}_{+}^{n}=[0, \infty)^{n}, \quad \mathbf{R}_{++}^{n}=(0, \infty)^{n}, \\
\Omega^{n}=\left\{\left(x_{1}, \ldots, x_{n}\right)^{\dagger} \in \mathbf{R}^{n} \mid 0 \leq x_{1}<x_{2}<\cdots<x_{n}\right\},
\end{gathered}
$$

and

$$
\bar{\Omega}^{n}=\left\{\left(x_{1}, \ldots, x_{n}\right)^{\dagger} \in \mathbf{R}^{n} \mid 0=x_{1}<x_{2}<\cdots<x_{n}\right\} .
$$

Another convenient notation has to do with the substitution of a component of a vector $\mathbf{x}=\left(x_{1}, \ldots, x_{n}\right)^{\dagger}$. Suppose the $j$-th component of $\mathbf{x}$ is replaced by $u$, 
we will denote the subsequent vector by $\mathbf{x}^{(j)}(u)$, that is,

$$
\mathbf{x}^{(j)}(u)=\left(x_{1}, \ldots, x_{j-1}, u, x_{j+1}, \ldots, x_{n}\right)^{\dagger} .
$$

Given $\mathbf{x} \in \mathbf{R}^{n}$, recall that the Vandermonde determinant in $\mathbf{x}$ is defined by

$$
\begin{aligned}
v_{n}(\mathbf{x}) & :=\operatorname{det}\left(x_{j}^{i-1}\right)_{n \times n} \\
& =\left|\begin{array}{ccccc}
1 & 1 & 1 & \ldots & 1 \\
x_{1} & x_{2} & x_{3} & \ldots & x_{n} \\
x_{1}^{2} & x_{2}^{2} & x_{3}^{2} & \ldots & x_{n}^{2} \\
\vdots & \vdots & \vdots & \ddots & \vdots \\
x_{1}^{n-1} & x_{2}^{n-1} & x_{3}^{n-1} & \ldots & x_{n}^{n-1}
\end{array}\right|=\prod_{1 \leq i<j \leq n}\left(x_{j}-x_{i}\right) .
\end{aligned}
$$

Given $\mathbf{x} \in \mathbf{R}^{n}$ and $\alpha \in \mathbf{R}^{n}$, we may extend the definition of Vandermonde determinant as follows:

$$
\begin{aligned}
V_{n}(\mathbf{x}, \alpha) & :=\operatorname{det}\left(x_{j}^{\alpha_{i}}\right)_{n \times n} \\
& =\left|\begin{array}{ccccc}
x_{1}^{\alpha_{1}} & x_{2}^{\alpha_{1}} & x_{3}^{\alpha_{1}} & \cdots & x_{n}^{\alpha_{1}} \\
x_{1}^{\alpha_{2}} & x_{2}^{\alpha_{2}} & x_{3}^{\alpha_{2}} & \cdots & x_{n}^{\alpha_{2}} \\
\vdots & \vdots & \vdots & \ddots & \vdots \\
x_{1}^{\alpha_{n}} & x_{2}^{\alpha_{n}} & x_{3}^{\alpha_{n}} & \cdots & x_{n}^{\alpha_{n}}
\end{array}\right| .
\end{aligned}
$$

In the above we need to make sure that each entry of the determinant is well defined. Such is the case when $x_{j} \geq 0$ and $\alpha_{i} \geq 0$, where $0^{0}=1$.

By means of these notations, given $\mathbf{d}_{1}, \ldots, \mathbf{d}_{n} \in \mathbf{R}^{m}$ and $\alpha \in \bar{\Omega}^{n}$, a generalized $\alpha$-polynomial is a function $\mathbf{f}:[0, \infty) \rightarrow \mathbf{R}^{m}$ defined by

$$
\mathbf{f}(u)=\sum_{j=1}^{n} \mathbf{d}_{j} u^{\alpha_{j}}, u \in[0, \infty),
$$

where we have employed the fact that $0^{0}=1$. Given $\mathbf{x} \in \Omega^{n}$ and $\mathbf{y}_{1}$, $\mathbf{y}_{2}, \ldots, \mathbf{y}_{n} \in \mathbf{R}^{m}$, if we try to find a generalized $\alpha$-polynomial, where $\alpha \in \bar{\Omega}^{n}$, that satisfies

$$
\mathbf{f}\left(x_{i}\right)=\mathbf{y}_{i}, i=1,2, \ldots, n,
$$


then we are led to a linear system of equations in the variables $\mathbf{d}_{1}, \ldots, \mathbf{d}_{n}$. Solving this system of vector equations, we easily see that

$$
V_{n}(\mathbf{x}, \alpha) \mathbf{f}(u)=\sum_{j=1}^{n} V_{n}\left(\mathbf{x}^{(j)}(u), \alpha\right) \mathbf{y}_{j} .
$$

Since $V_{n}(\mathbf{x}, \alpha)>0$ for $\mathbf{x} \in \Omega^{n}$ and $\alpha \in \bar{\Omega}^{n}$ (see [3, p. 212, Theorem 1]), we see further that the desired $\alpha$-polynomial satisfying (4) can be expressed as

$$
\mathbf{f}(u)=\sum_{j=1}^{n} \frac{V_{n}\left(\mathbf{x}^{(j)}(u), \alpha\right)}{V_{n}(\mathbf{x}, \alpha)} \mathbf{y}_{j}, u \in[0, \infty) .
$$

Now that the existence and uniqueness of the desired interpolating polynomial is out of the way, the main theorem to be shown will be the following.

Theorem 1. Given $\mathbf{x} \in \Omega^{n}$ and $\alpha \in \bar{\Omega}^{n}$ as well as $\mathbf{y}_{1}, \ldots, \mathbf{y}_{n} \in \mathbf{R}^{m}$. The generalized interpolating $\alpha$-polynomial $\mathbf{f}$ in (5) will satisfy

$$
\begin{aligned}
\|\mathbf{f}(u)-\overline{\mathbf{y}}\| \leq & {\left[1+\frac{2}{n(n-1)}\left(\sum_{i=0}^{\left[\frac{n-1}{2}\right]\left[\frac{n-1}{2}\right]} \sum_{j=1}^{1} \frac{1}{i+j}-\sum_{j=1}^{n-1} \frac{1}{j}\right)\right]^{\frac{n(n-1)}{2}} } \\
& \times \frac{v_{n}\left(\frac{\max _{1 \leq i \leq n-1}\left\{x_{i+1}^{\theta}-x_{i}^{\theta}\right\}}{\theta x_{n}^{\theta}} \alpha\right)}{V_{n}\left(\frac{\mathbf{x}}{x_{n}}, \alpha\right)} \sum_{j=1}^{n}\left\|\mathbf{y}_{j}-\overline{\mathbf{y}}\right\|
\end{aligned}
$$

for any $u \in\left[x_{1}, x_{n}\right]$ and any $\overline{\mathbf{y}} \in \mathbf{R}^{m}$, where $\theta=\min _{1 \leq i \leq n-1} \Delta \alpha_{i}$.

In the above and later discussions, we employ the greatest integer function [·].

\section{Preparatory lemmas}

We first recall the following result, which was already used in deriving the $\alpha$ interpolating polynomial.

Lemma 1. ([3, p. 212, Theorem 1]). Let $\mathbf{x} \in \Omega^{n}$ and $\alpha \in \bar{\Omega}^{n}$. Then $V_{n}(\mathbf{x}, \alpha)>0$. 
Lemma 2. ([5, p. 1047, Lemma 2.2]). Let $\mathbf{x}, \alpha \in \Omega^{n}$ and $\beta=\left(\beta_{1}, \beta_{2}, \ldots, \beta_{n-1}\right)$ where $\beta_{j}=\alpha_{j+1}-\alpha_{1}-1$ for $j=1,2, \ldots, n-1$. Then

$$
\begin{aligned}
V_{n}(\mathbf{x}, \alpha)= & \left(\prod_{j=1}^{n} x_{j}\right)^{\alpha_{1}}\left[\prod_{j=2}^{n}\left(\alpha_{j}-\alpha_{1}\right)\right] \\
& \times \int_{x_{1}}^{x_{2}} d t_{1} \int_{x_{2}}^{x_{3}} d t_{2} \cdots \int_{x_{n-1}}^{x_{n}} d t_{n-1} V_{n-1}(\mathbf{t}, \beta),
\end{aligned}
$$

where $\mathbf{t}=\left(t_{1}, t_{2}, \ldots, t_{n-1}\right)$.

Lemma 3. Let $\mathbf{x}, \alpha \in \Omega_{n}$. If $\Delta \alpha_{j} \geq 1$ for $j=1,2, \ldots, n-1$, then

$$
V_{n}(\mathbf{x}, \alpha) \leq\left(\prod_{j=1}^{n-1} j !\right)^{-1} v_{n}(\alpha) v_{n}(\mathbf{x})\left(\frac{x_{n-1}^{d_{n}}+x_{n}^{d_{n}}}{2}\right)^{\frac{\sum_{j=1}^{n} \alpha_{j}-2^{-1} n(n-1)}{d_{n}}},
$$

where $d_{n}=\max \left\{1, \alpha_{n}-\alpha_{n-1}-1\right\}$.

Proof. When $n=2$, we may see from (7) that

$$
V_{2}(\mathbf{x}, \alpha)=\left(x_{1} x_{2}\right)^{\alpha_{1}}\left(\alpha_{2}-\alpha_{1}\right) \int_{x_{1}}^{x_{2}} t_{1}^{\alpha_{2}-\alpha_{1}-1} d t_{1} .
$$

If $0 \leq \alpha_{2}-\alpha_{1}-1 \leq 1$, then $d_{2}=1$ and the function $q(t)=t^{\alpha_{2}-\alpha_{1}-1}$ is concave over the interval $\left[x_{1}, x_{2}\right]$. In view of the Hadamard's inequality (see e.g. [2]) and the A-G inequality, we see that

$$
\begin{aligned}
V_{2}(\mathbf{x}, \alpha) & =\left(x_{1} x_{2}\right)^{\alpha_{1}}\left(\alpha_{2}-\alpha_{1}\right) \int_{x_{1}}^{x_{2}} t_{1}^{\alpha_{2}-\alpha_{1}-1} d t_{1} \\
& \leq\left(x_{1} x_{2}\right)^{\alpha_{1}}\left(\alpha_{2}-\alpha_{1}\right)\left(x_{2}-x_{1}\right)\left(\frac{x_{1}+x_{2}}{2}\right)^{\alpha_{2}-\alpha_{1}-1} \\
& \leq\left(\frac{x_{1}+x_{2}}{2}\right)^{2 \alpha_{1}}\left(\alpha_{2}-\alpha_{1}\right)\left(x_{2}-x_{1}\right)\left(\frac{x_{1}+x_{2}}{2}\right)^{\alpha_{2}-\alpha_{1}-1} \\
& =v_{2}(\alpha) v_{2}(\mathbf{x})\left(\frac{x_{1}^{d_{2}}+x_{2}^{d_{2}}}{2}\right)^{\frac{\alpha_{1}+\alpha_{2}-1}{d_{2}}} .
\end{aligned}
$$


If $\alpha_{2}-\alpha_{1}-1>1$, then $d_{2}=\alpha_{2}-\alpha_{1}-1$ and the function $q(t)=t^{\alpha_{2}-\alpha_{1}-1}$ is convex over the interval $\left[x_{1}, x_{2}\right]$. Again, from the Hadamard's inequality and the A-G inequality,

$$
\begin{aligned}
V_{2}(\mathbf{x}, \alpha) & =\left(x_{1} x_{2}\right)^{\alpha_{1}}\left(\alpha_{2}-\alpha_{1}\right) \int_{x_{1}}^{x_{2}} t_{1}^{\alpha_{2}-\alpha_{1}-1} d t_{1} \\
& <\left(x_{1} x_{2}\right)^{\alpha_{1}}\left(\alpha_{2}-\alpha_{1}\right)\left(x_{2}-x_{1}\right) \frac{x_{1}^{d_{2}}+x_{2}^{d_{2}}}{2} \\
& \leq\left(\frac{x_{1}^{d_{2}}+x_{2}^{d_{2}}}{2}\right)^{\frac{2 \alpha_{1}}{d_{2}}}\left(\alpha_{2}-\alpha_{1}\right)\left(x_{2}-x_{1}\right) \frac{x_{1}^{d_{2}}+x_{2}^{d_{2}}}{2} \\
& =v_{2}(\alpha) v_{2}(\mathbf{x})\left(\frac{x_{1}^{d_{2}}+x_{2}^{d_{2}}}{2}\right)^{\frac{\alpha_{1}+\alpha_{2}-1}{d_{2}}} .
\end{aligned}
$$

These show that (8) is valid for $n=2$.

We now assume by induction that (8) holds when $n$ is replaced by $n-1$ (where $n-1 \geq 2$ ). By our assumption that $\Delta \alpha_{j} \geq 1$ for $j=1,2, \ldots, n-1$, we see that

$$
\begin{gathered}
0 \leq \beta_{1}<\beta_{2}<\cdots<\beta_{n-1}, \\
\beta_{j+1}-\beta_{j}=\alpha_{j+2}-\alpha_{j+1} \geq 1, j=1,2, \ldots, n-2, \\
d_{n-1}^{*}:=\max \left\{1, \beta_{n-1}-\beta_{n-2}-1\right\} \\
\quad=\max \left\{1, \alpha_{n}-\alpha_{n-1}-1\right\}=d_{n},
\end{gathered}
$$

and

$$
\begin{aligned}
& \sum_{j=1}^{n-1}\left(\alpha_{j+1}-\alpha_{1}-1\right)-2^{-1}(n-1)(n-2) \\
= & \sum_{j=1}^{n-1}\left[\sum_{r=1}^{j}\left(\alpha_{r+1}-\alpha_{r}\right)-1\right]-2^{-1}(n-1)(n-2) \\
\geq & \sum_{j=1}^{n-1}(j-1)-2^{-1}(n-1)(n-2)=0 .
\end{aligned}
$$


Hence,

$$
\begin{aligned}
& V_{n-1}(\mathbf{t}, \beta) \\
& \leq\left(\prod_{j=1}^{n-2} j !\right)^{-1} v_{n-1}(\beta) v_{n-1}(\mathbf{t})\left(\frac{d_{n-2}^{*}+t_{n-1}^{*}}{2}\right)^{\frac{d_{n-1}^{*}}{n-1} \beta_{j}-2^{-1}(n-1)(n-2)}
\end{aligned}
$$

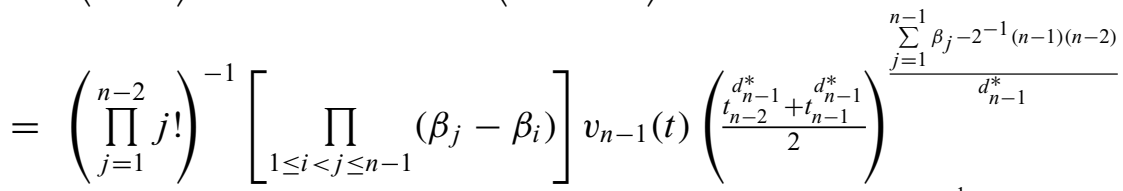

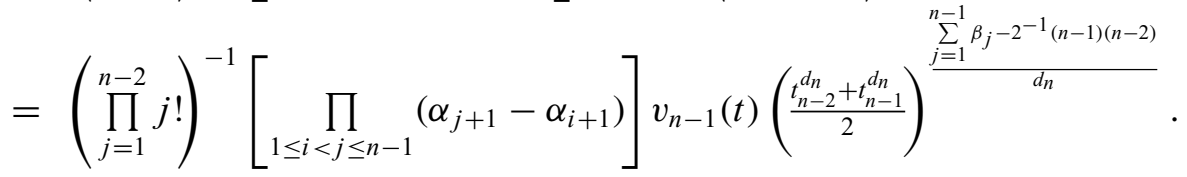

Furthermore, if $x_{j}<t_{j}<x_{j+1}$ for $j=1,2, \ldots, n-1$, then

$$
\begin{aligned}
V_{n-1}(\mathbf{t}, \beta) \leq & \left(\prod_{j=1}^{n-2} j !\right)^{-1}\left[\prod_{2 \leq i<j \leq n}\left(\alpha_{j}-\alpha_{i}\right)\right] \\
& \times v_{n-1}(\mathbf{t})\left(\frac{x_{n-1}^{d_{n}}+x_{n}^{d_{n}}}{2}\right) .
\end{aligned}
$$

Next, if we take $\alpha=(0,1,2, \ldots, n-1)$ in (7), we see that

$$
v_{n}(\mathbf{x})=\left[\prod_{j=2}^{n}(j-1)\right] \int_{x_{1}}^{x_{2}} d t_{1} \int_{x_{2}}^{x_{3}} d t_{2} \cdots \int_{x_{n-1}}^{x_{n}} d t_{n-1} v_{n-1}(\mathbf{t}),
$$

and hence

$$
\begin{aligned}
& \int_{x_{1}}^{x_{2}} d t_{1} \int_{x_{2}}^{x_{3}} d t_{2} \cdots \int_{x_{n-1}}^{x_{n}} d t_{n-1} v_{n-1}(\mathbf{t}) \\
= & \frac{1}{(n-1) !} v_{n}(\mathbf{x})=\frac{1}{(n-1) !} \prod_{1 \leq i<j \leq n}\left(x_{j}-x_{i}\right) .
\end{aligned}
$$


With the above information at hand, we may now see that

$$
\begin{aligned}
& V_{n}(\mathbf{x}, \alpha) \\
= & \left(\prod_{j=1}^{n} x_{j}\right)^{\alpha_{1}}\left[\prod_{j=2}^{n}\left(\alpha_{j}-\alpha_{1}\right)\right] \int_{x_{1}}^{x_{2}} d t_{1} \int_{x_{2}}^{x_{3}} d t_{2} \ldots \int_{x_{n-1}}^{x_{n}} d t_{n-1} V_{n-1}(\mathbf{t}, \beta) \\
\leq & \left(\prod_{j=1}^{n} x_{j}\right)^{\alpha_{1}}\left[\prod_{j=2}^{n}\left(\alpha_{j}-\alpha_{1}\right)\right]\left[\int_{x_{1}}^{x_{2}} d t_{1} \int_{x_{2}}^{x_{3}} d t_{2} \cdots \int_{x_{n-1}}^{x_{n}} d t_{n-1} v_{n-1}(\mathbf{t})\right] \\
& \times\left[\left(\prod_{j=1}^{n-2} j !\right)^{-1} \prod_{2 \leq i<j \leq n}\left(\alpha_{j}-\alpha_{i}\right)\right]\left(\frac{x_{n-1}^{d_{n}+x_{n}^{d_{n}}}}{2}\right)^{\frac{\sum_{j=1}^{n-1} \beta_{j}-2^{-1}(n-1)(n-2)}{d_{n}}} \\
= & \left(\prod_{j=1}^{n-2} j !\right)^{-1}\left(\prod_{j=1}^{n} x_{j}\right)^{\alpha_{1}}\left[\prod_{1 \leq i<j \leq n}\left(\alpha_{j}-\alpha_{i}\right)\right][(n-1) !]^{-1} \\
& \times v_{n}(\mathbf{x})\left(\frac{x_{n-1}^{d_{n}+x_{n}^{d_{n}}}}{2}\right)^{\frac{\sum_{j=1}^{n-1} \beta_{j}-2^{-1}(n-1)(n-2)}{d_{n}}}, \\
= & \left(\prod_{j=1}^{n-1} j !\right)^{-1}\left(\prod_{j=1}^{n} x_{j}\right)^{\alpha_{1}} v_{n}(\alpha) v_{n}(\mathbf{x})\left(\frac{x_{n-1}^{d_{n}+x_{n}^{d_{n}}}}{2}\right)^{\frac{\sum_{j=1}^{n-1} \beta_{j}-2^{-1}(n-1)(n-2)}{d_{n}}}
\end{aligned}
$$

that is,

$$
\begin{gathered}
V_{n}(\mathbf{x}, \alpha) \\
\leq\left(\prod_{j=1}^{n-1} j !\right)^{-1}\left(\prod_{j=1}^{n} x_{j}\right)^{\alpha_{1}} v_{n}(\alpha) v_{n}(\mathbf{x})\left(\frac{x_{n-1}^{d_{n}}+x_{n}^{d_{n}}}{2}\right)^{\frac{\sum_{j=1}^{n-1} \beta_{j}-2^{-1}(n-1)(n-2)}{d_{n}}} .
\end{gathered}
$$

Since $\mathbf{x} \in \Omega^{n}$ and $d_{n} \geq 1$, we also have

$$
\begin{gathered}
x_{j}<\left(\frac{x_{n-1}^{d_{n}}+x_{n}^{d_{n}}}{2}\right)^{\frac{1}{d_{n}}}, j=1,2, \ldots, n-1, \\
x_{n-1} x_{n}<\left(\frac{x_{n-1}^{d_{n}}+x_{n}^{d_{n}}}{2}\right)^{\frac{2}{d_{n}}}
\end{gathered}
$$


and

$$
\left(\prod_{j=1}^{n} x_{j}\right)^{\alpha_{1}} \leq\left[\left(\frac{x_{n-1}^{d_{n}}+x_{n}^{d_{n}}}{2}\right)^{\frac{n-2}{d_{n}}}\left(\frac{x_{n-1}^{d_{n}}+x_{n}^{d_{n}}}{2}\right)^{\frac{2}{d_{n}}}\right]^{\alpha_{1}}=\left(\frac{x_{n-1}^{d_{n}}+x_{n}^{d_{n}}}{2}\right)^{\frac{n \alpha_{1}}{d_{n}}} .
$$

Thus from (14), we may further assert that

$$
\begin{aligned}
& V_{n}(\mathbf{x}, \alpha) \\
& \leq\left(\prod_{j=1}^{n-1} j !\right)^{-1}\left(\prod_{j=1}^{n} x_{j}\right)^{\alpha_{1}} v_{n}(\alpha) v_{n}(\mathbf{x})\left(\frac{x_{n-1}^{d_{n}}+x_{n}^{d_{n}}}{2}\right)^{\frac{\sum_{j=1}^{n-1}\left(\alpha_{j+1}-\alpha_{1}\right)-2^{-1} n(n-1)}{d_{n}}} \\
& \leq\left(\prod_{j=1}^{n-1} j !\right)^{-1}\left(\frac{x_{n-1}^{d_{n}}+x_{n}^{d_{n}}}{2}\right)^{\frac{n \alpha_{1}}{d_{n}}} v_{n}(\alpha) v_{n}(\mathbf{x})\left(\frac{x_{n-1}^{d_{n}}+x_{n}^{d_{n}}}{2}\right)^{\frac{\sum_{j=1}^{n-1}\left(\alpha_{j+1}-\alpha_{1}\right)-2^{-1} n(n-1)}{d_{n}}} \\
& =\left(\prod_{j=1}^{n-1} j !\right)^{-1} v_{n}(\alpha) v_{n}(\mathbf{x})\left(\frac{x_{n-1}^{d_{n}}+x_{n}^{d_{n}}}{2}\right)^{\frac{\sum_{j=1}^{n} \alpha_{j}-2^{-1} n(n-1)}{d_{n}}} .
\end{aligned}
$$

The proof is complete.

Lemma 4. ([5, p. 1050, Lemma 2.4]). Let $\mathbf{x} \in \mathbf{R}^{n}$. Then

$$
\sum_{1 \leq i<j \leq n} \frac{x_{j}-x_{i}}{j-i}=\sum_{k=1}^{n-1}\left(\sum_{i=0}^{k-1} \sum_{j=1}^{n-k} \frac{1}{i+j}\right) \Delta x_{k} .
$$

Lemma 5. ([5, p. 1051, Lemma 2.5]). Let

$$
\lambda_{k}:=\sum_{i=0}^{k-1} \sum_{j=1}^{n-k} \frac{1}{i+j}, k=1,2, \ldots, n-1 .
$$

Then

$$
\sum_{k=1}^{n-1} \lambda_{k}=\frac{n(n-1)}{2}
$$

and

$$
\lambda_{1} \leq \lambda_{2} \leq \cdots \leq \lambda_{\left[\frac{n+1}{2}\right]-1} \leq \lambda_{\left[\frac{n+1}{2}\right]} \geq \lambda_{\left[\frac{n+1}{2}\right]+1} \geq \cdots \geq \lambda_{n-2} \geq \lambda_{n-1}=\lambda_{1} .
$$


Lemma 6. Let $\mathbf{x} \in \Omega^{n}$. Then for $u \in\left[x_{1}, x_{n}\right]$ and $r \in\{1,2, \ldots, n\}$,

$$
\begin{aligned}
\left|v_{n}\left(\mathbf{x}^{(r)}(u)\right)\right| \leq & \left(\prod_{j=1}^{n-1} j !\right)\left[1+\frac{2}{n(n-1)}\left(\sum_{i=0}^{\left[\frac{n-1}{2}\right]\left[\frac{n-1}{2}\right]} \sum_{j=1} \frac{1}{i+j}-\sum_{j=1}^{n-1} \frac{1}{j}\right)\right]^{\frac{n(n-1)}{2}} \\
& \times\left(\max _{1 \leq i \leq n-1} \Delta x_{i}\right)^{\frac{n(n-1)}{2}} .
\end{aligned}
$$

Proof. Since $u \in\left[x_{1}, x_{n}\right]$, there is $s \in\{1,2, \ldots, n-1\}$ such that $x_{s} \leq u \leq$ $x_{s+1}$. Let us move the $r$-th column of the determinant $v_{n}\left(\mathbf{x}^{(r)}(u)\right)$ and 'insert' it between the $s$-th and the $(s+1)$-th column of $v_{n}\left(\mathbf{x}^{(r)}(u)\right)$. The resulting determinant will be denoted by $v_{n}\left(\mathbf{x}^{*(r)}(u)\right)$, where

$$
\begin{aligned}
\mathbf{x}^{*(r)}(u) & =\left(x_{1}^{*(r)}(u), x_{2}^{*(r)}(u), \ldots, x_{n}^{*(r)}(u)\right)^{\dagger} \\
& = \begin{cases}\left(x_{1}, \ldots, x_{r-1}, x_{r+1}, \ldots, x_{s}, u, x_{s+1}, \ldots, x_{n}\right)^{\dagger}, & s \geq r+1 ; \\
\left(x_{1}, \ldots, x_{r-1}, u, x_{r+1}, \ldots, x_{n}\right)^{\dagger}, & s=r-1, r ; \\
\left(x_{1}, \ldots, x_{s}, u, x_{s+1}, \ldots, x_{r-1}, x_{r+1}, \ldots, x_{n}\right)^{\dagger}, & s \leq r-2 .\end{cases}
\end{aligned}
$$

Then $v_{n}\left(\mathbf{x}^{*(r)}(u)\right) \geq 0$. Furthermore, in view of the A-G inequality [6-9] and (15),

$$
\begin{aligned}
\left|v_{n}\left(\mathbf{x}^{(r)}(u)\right)\right| & =v_{n}\left(\mathbf{x}^{*(r)}(u)\right) \\
& =\prod_{1 \leq i<j \leq n}\left(x_{j}^{*(r)}(u)-x_{i}^{*(r)}(u)\right) \\
& =\prod_{1 \leq i<j \leq n}(j-i) \prod_{1 \leq i<j \leq n} \frac{x_{j}^{*(r)}(u)-x_{i}^{*(r)}(u)}{j-i} \\
& =\left(\prod_{j=1}^{n-1} j !\right) \prod_{1 \leq i<j \leq n} \frac{x_{j}^{*(r)}(u)-x_{i}^{*(r)}(u)}{j-i} \\
& \leq\left(\prod_{j=1}^{n-1} j !\right)\left[\frac{2}{n(n-1)} \sum_{1 \leq i<j \leq n} \frac{x_{j}^{*(r)}(u)-x_{i}^{*(r)}(u)}{j-i}\right]^{\frac{n(n-1)}{2}} \\
& =\left(\prod_{j=1}^{n-1} j !\right)\left[\frac{2}{n(n-1)} \sum_{k=1}^{n-1}\left(\sum_{i=0}^{k-1} \sum_{j=1}^{n-k} \frac{1}{i+j}\right) \Delta x_{k}^{*(r)}(u)\right]^{\frac{n(n-1)}{2}} \\
& \leq\left(\prod_{j=1}^{n-1} j !\right)\left[\frac{2}{n(n-1)} \sum_{k=1}^{n-1} \lambda_{k} \Delta x_{k}^{*(r)}(u)\right]^{\frac{n(n-1)}{2}},
\end{aligned}
$$


where $\lambda_{k}$ is defined in Lemma 5. Next we assert that

$$
\sum_{k=1}^{n-1} \lambda_{k} \Delta x_{k}^{*(r)}(u) \leq\left[\frac{n(n-1)}{2}+\sum_{i=0}^{\left[\frac{n-1}{2}\right]} \sum_{j=1}^{\left[\frac{n-1}{2}\right]} \frac{1}{i+j}-\sum_{j=1}^{n-1} \frac{1}{j}\right] \max _{1 \leq i \leq n-1} \Delta x_{i} .
$$

Indeed, if $s=r-1$ or $s=r$, then

$$
\mathbf{x}^{*(r)}(u)=\mathbf{x}^{(r)}(u)
$$

If $2 \leq r \leq n-1$, then from Lemma 5 ,

$$
\begin{aligned}
& \sum_{k=1}^{n-1} \lambda_{k} \Delta x_{k}^{*(r)}(u) \\
& =\sum_{k \in\{1, \ldots, n-1\} \backslash\{r-1, r\}} \lambda_{k} \Delta x_{k}^{(r)}(u)+\lambda_{r-1} \Delta x_{r-1}^{(r)}(u)+\lambda_{r} \Delta x_{r}^{(r)}(u) \\
& =\sum_{k \in\{1, \ldots, n-1\} \backslash\{r-1, r\}} \lambda_{k} \Delta x_{k}+\lambda_{r-1}\left(u-x_{r-1}\right)+\lambda_{r}\left(x_{r+1}-u\right) \\
& \leq \sum_{k \in\{1, \ldots, n-1\} \backslash\{r-1, r\}} \lambda_{k} \Delta x_{k}+\max \left\{\lambda_{r-1}, \lambda_{r}\right\}\left(u-x_{r-1}\right) \\
& +\max \left\{\lambda_{r-1}, \lambda_{r}\right\}\left(x_{r+1}-u\right) \\
& =\sum_{k \in\{1, \ldots, n-1\} \backslash\{r-1, r\}} \lambda_{k} \Delta x_{k}+\max \left\{\lambda_{r-1}, \lambda_{r}\right\}\left(x_{r+1}-x_{r-1}\right) \\
& \leq \sum_{k \in\{1, \ldots, n-1\} \backslash\{r-1, r\}} \lambda_{k} \max _{1 \leq i \leq n-1} \Delta x_{i}+2 \max \left\{\lambda_{r-1}, \lambda_{r}\right\} \max _{1 \leq i \leq n-1} \Delta x_{i} \\
& =\left(\sum_{k \in\{1, \ldots, n-1\} \backslash\{r-1, r\}} \lambda_{k}+2 \max \left\{\lambda_{r-1}, \lambda_{r}\right\}\right) \max _{1 \leq i \leq n-1} \Delta x_{i} \\
& =\left(\sum_{k=1}^{n-1} \lambda_{k}+\max _{1 \leq i \leq n-1}\left\{\lambda_{r-1}, \lambda_{r}\right\}-\min _{1 \leq i \leq n-1}\left\{\lambda_{r-1}, \lambda_{r}\right\}\right) \max _{1 \leq i \leq n-1} \Delta x_{i} \\
& \leq\left(\sum_{k=1}^{n-1} \lambda_{k}+\max _{1 \leq i \leq n-1}\left\{\lambda_{i}\right\}-\min _{1 \leq i \leq n-1}\left\{\lambda_{i}\right\}\right) \max _{1 \leq i \leq n-1} \Delta x_{i}
\end{aligned}
$$




$$
\begin{aligned}
& =\left(\sum_{k=1}^{n-1} \lambda_{k}+\lambda_{\left[\frac{n+1}{2}\right]}-\lambda_{1}\right) \max _{1 \leq i \leq n-1} \Delta x_{i} \\
& =\left[\frac{n(n-1)}{2}+\sum_{i=0}^{\left[\frac{n-1}{2}\right]} \sum_{j=1}^{\left[\frac{n-1}{2}\right]} \frac{1}{i+j}-\sum_{j=1}^{n-1} \frac{1}{j}\right] \max _{1 \leq i \leq n-1} \Delta x_{i} .
\end{aligned}
$$

If $r=1$, then

$$
\begin{aligned}
\sum_{k=1}^{n-1} \lambda_{k} \Delta x_{k}^{*(r)}(u) & =\sum_{2 \leq k \leq n-1} \lambda_{k} \Delta x_{k}^{(1)}(u)+\lambda_{1} \Delta x_{1}^{(1)}(u) \\
& =\sum_{2 \leq k \leq n-1} \lambda_{k} \Delta x_{k}+\lambda_{1}\left(x_{2}-u\right) \\
& \leq \sum_{2 \leq k \leq n-1} \lambda_{k} \max _{1 \leq i \leq n-1} \Delta x_{i}+\lambda_{1} \max _{1 \leq i \leq n-1} \Delta x_{i} \\
& =\frac{n(n-1)}{2} \max _{1 \leq i \leq n-1} \Delta x_{i} \\
& \leq\left[\frac{n(n-1)}{2}+\sum_{i=0}^{\left[\frac{n-1}{2}\right]\left[\frac{n-1}{2}\right]} \frac{1}{i+j}-\sum_{j=1}^{n-1} \frac{1}{j}\right] \max _{1 \leq i \leq n-1} \Delta x_{i}
\end{aligned}
$$

If $r=n$, then

$$
\begin{aligned}
\sum_{k=1}^{n-1} \lambda_{k} \Delta x_{k}^{*(r)}(u) & =\sum_{1 \leq k \leq n-2} \lambda_{k} \Delta x_{k}^{(n)}(u)+\lambda_{n-1} \Delta x_{n-1}^{(n)}(u) \\
& =\sum_{1 \leq k \leq n-2} \lambda_{k} \Delta x_{k}+\lambda_{n-1}\left(u-x_{n-1}\right) \\
& \leq \sum_{1 \leq k \leq n-2} \lambda_{k} \max _{1 \leq i \leq n-1} \Delta x_{i}+\lambda_{n-1} \max _{1 \leq i \leq n-1} \Delta x_{i} \\
& =\frac{n(n-1)}{2} \max _{1 \leq i \leq n-1} \Delta x_{i} \\
& \leq\left[\frac{n(n-1)}{2}+\sum_{i=0}^{\left[\frac{n-1}{2}\right]\left[\frac{n-1}{2}\right]} \frac{1}{i+j}-\sum_{j=1}^{n-1} \frac{1}{j}\right] \max _{1 \leq i \leq n-1} \Delta x_{i}
\end{aligned}
$$


Next, suppose $s \leq r-2$ or $s \geq r+1$. Let

$$
p=\left\{\begin{array}{cl}
r-1, & \text { if } s \geq r+1 \\
r, & \text { if } s \leq r-2
\end{array} \quad \text { and } \quad q=\left\{\begin{array}{cl}
s-1, & \text { if } s \geq r+1 \\
s, & \text { if } s \leq r-2
\end{array} .\right.\right.
$$

Since $1 \leq r \leq n$ and $1 \leq s \leq n-1$, we have $1 \leq q \leq n-2$. If $2 \leq r \leq$ $n-1$, then $1 \leq p \leq n-1$, and hence by Lemma 5 ,

$$
\begin{aligned}
& \sum_{k=1}^{n-1} \lambda_{k} \Delta x_{k}^{*(r)}(u) \\
& =\sum_{k \in\{1, \ldots, n-1\} \backslash\{p, q, q+1\}} \lambda_{k} \Delta x_{k}^{*(r)}(u)+\lambda_{p} \Delta x_{p}^{*(r)}(u)+\lambda_{q} \Delta x_{q}^{*(r)}(u) \\
& +\lambda_{q+1} \Delta x_{q+1}^{*(r)}(u) \\
& =\sum_{k \in\{1, \ldots, n-1\} \backslash\{p, q, q+1\}} \lambda_{k} \Delta x_{k}+\lambda_{p}\left(x_{r+1}-x_{r-1}\right)+\lambda_{q}\left(u-x_{s}\right) \\
& +\lambda_{q+1}\left(x_{s+1}-u\right) \\
& \leq \sum_{k \in\{1, \ldots, n-1\} \backslash\{p, q, q+1\}} \lambda_{k} \Delta x_{k}+\lambda_{p}\left(x_{r+1}-x_{r-1}\right) \\
& +\max \left\{\lambda_{q}, \lambda_{q+1}\right\}\left(x_{s+1}-x_{s}\right) \\
& \leq \sum_{k \in\{1, \ldots, n-1\} \backslash\{p, q, q+1\}} \lambda_{k} \max _{1 \leq i \leq n-1} \Delta x_{i}+2 \lambda_{p} \max _{1 \leq i \leq n-1} \Delta x_{i} \\
& +\max \left\{\lambda_{q}, \lambda_{q+1}\right\} \max _{1 \leq i \leq n-1} \Delta x_{i} \\
& =\left(\sum_{k \in\{1, \ldots, n-1\} \backslash\{p, q, q+1\}} \lambda_{k}+2 \lambda_{p}+\max \left\{\lambda_{q}, \lambda_{q+1}\right\}\right) \max _{1 \leq i \leq n-1} \Delta x_{i} \\
& =\left(\sum_{k=1}^{n-1} \lambda_{k}+\lambda_{p}-\min _{1 \leq i \leq n-1}\left\{\lambda_{q}, \lambda_{q+1}\right\}\right) \max _{1 \leq i \leq n-1} \Delta x_{i} \\
& \leq\left(\sum_{k=1}^{n-1} \lambda_{k}+\max _{1 \leq i \leq n-1}\left\{\lambda_{i}\right\}-\min _{1 \leq i \leq n-1}\left\{\lambda_{i}\right\}\right) \max _{1 \leq i \leq n-1} \Delta x_{i} \\
& =\left[\frac{n(n-1)}{2}+\sum_{i=0}^{\left[\frac{n-1}{2}\right]} \sum_{j=1}^{\left[\frac{n-1}{2}\right]} \frac{1}{i+j}-\sum_{j=1}^{n-1} \frac{1}{j}\right] \max _{1 \leq i \leq n-1} \Delta x_{i} \text {. }
\end{aligned}
$$


If $r=1$ or $r=n$, from Lemma 5 ,

$$
\begin{aligned}
& \sum_{k=1}^{n-1} \lambda_{k} \Delta x_{k}^{*(r)}(u) \\
& =\sum_{k \in\{1, \ldots, n-1\} \backslash\{q, q+1\}} \lambda_{k} \Delta x_{k}^{*(r)}(u)+\lambda_{q} \Delta x_{q}^{*(r)}(u)+\lambda_{q+1} \Delta x_{q+1}^{*(r)}(u) \\
& =\sum_{k \in\{1, \ldots, n-1\} \backslash\{q, q+1\}} \lambda_{k} \Delta x_{k}+\lambda_{q}\left(u-x_{s}\right)+\lambda_{q+1}\left(x_{s+1}-u\right) \\
& \leq \sum_{k \in\{1, \ldots, n-1\} \backslash\{q, q+1\}} \lambda_{k} \Delta x_{k}+\max \left\{\lambda_{q}, \lambda_{q+1}\right\}\left(x_{s+1}-x_{s}\right) \\
& \leq \sum_{k \in\{1, \ldots, n-1\} \backslash\{q, q+1\}} \lambda_{k} \max _{1 \leq i \leq n-1}\left\{\Delta x_{i}\right\}+\max \left\{\lambda_{q}, \lambda_{q+1}\right\} \max _{1 \leq i \leq n-1} \Delta x_{i} \\
& =\left(\sum_{k=1}^{n-1} \lambda_{k}-\min \left\{\lambda_{q}, \lambda_{q+1}\right\}\right) \max _{1 \leq i \leq n-1} \Delta x_{i} \\
& \leq\left(\sum_{k=1}^{n-1} \lambda_{k}-\min _{1 \leq i \leq n-1}\left\{\lambda_{i}\right\}\right) \max _{1 \leq i \leq n-1} \Delta x_{i} \\
& =\left(\sum_{k=1}^{n-1} \lambda_{k}-\lambda_{1}\right) \max _{1 \leq i \leq n-1} \Delta x_{i} \\
& <\left[\frac{n(n-1)}{2}+\sum_{i=0}^{\left[\frac{n-1}{2}\right]\left[\frac{n-1}{2}\right]} \sum_{j=1}^{1+j}-\sum_{j=1}^{n-1} \frac{1}{j}\right] \max _{1 \leq i \leq n-1} \Delta x_{i} .
\end{aligned}
$$

The inequality (20) is thus proved.

By combining (19) and (20), we see that

$$
\begin{aligned}
\left|v_{n}\left(\mathbf{x}^{(r)}(u)\right)\right| \leq & \left(\prod_{j=1}^{n-1} j !\right)\left[\frac{2}{n(n-1)} \sum_{k=1}^{n-1} \lambda_{k} \Delta x_{k}^{*(r)}(u)\right]^{\frac{n(n-1)}{2}} \\
\leq & \left(\prod_{j=1}^{n-1} j !\right)\left[1+\frac{2}{n(n-1)}\left(\sum_{i=0}^{\left[\frac{n-1}{2}\right]\left[\frac{n-1}{2}\right]} \frac{1}{j=1}-\sum_{j=1}^{n-1} \frac{1}{j}\right)\right]^{\frac{n(n-1)}{2}} \\
& \times\left(\max _{1 \leq i \leq n-1} \Delta x_{i}\right)^{\frac{n(n-1)}{2}} .
\end{aligned}
$$

The proof of (18) is complete. 
Lemma 7. Let $\mathbf{x}, \alpha \in \Omega^{n}$ such that $\Delta \alpha_{j} \geq 1$ for $j=1,2, \ldots, n-1$. Then for any $u \in\left[x_{1}, x_{n}\right]$ and $j \in\{1,2, \ldots, n\}$,

$$
\begin{aligned}
\left|V_{n}\left(\mathbf{x}^{(j)}(u), \alpha\right)\right| \leq & {\left[1+\frac{2}{n(n-1)}\left(\sum_{i=0}^{\left[\frac{n-1}{2}\right]\left[\frac{n-1}{2}\right]} \sum_{j=1}^{1} \frac{1}{i+j}-\sum_{j=1}^{n-1} \frac{1}{j}\right)\right]^{\frac{n(n-1)}{2}} } \\
& \times v_{n}\left(\frac{\max _{1 \leq i \leq n-1} \Delta x_{i}}{x_{n}} \alpha\right) x_{n}^{\sum_{j=1}^{n} \alpha_{j}} .
\end{aligned}
$$

Proof. Since $x_{1} \leq u \leq x_{n}$, there is $s \in\{1,2, \ldots, n-1\}$ such that $0 \leq x_{s} \leq$ $u \leq x_{s+1}$. Let us move the $j$-th column of the determinant $V_{n}\left(\mathbf{x}^{(r)}(u), \alpha\right)$ and 'insert' it between the $s$-th and the $(s+1)$-th columns of $V_{n}\left(\mathbf{x}^{(r)}(u), \alpha\right)$. The resulting determinant will be denoted by $V_{n}\left(\mathbf{x}^{*(r)}(u), \alpha\right)$ where

$$
\begin{aligned}
\mathbf{x}^{*(j)}(u) & =\left(x_{1}^{*(j)}(u), x_{2}^{*(j)}(u), \ldots, x_{n}^{*(j)}(u)\right)^{\dagger} \\
& = \begin{cases}\left(x_{1}, \ldots, x_{j-1}, x_{j+1}, \ldots, x_{s}, u, x_{s+1}, \ldots, x_{n}\right)^{\dagger}, & s \geq j+1, \\
\left(x_{1}, \ldots, x_{j-1}, u, x_{j+1}, \ldots, x_{n}\right)^{\dagger}, & s=j-1, j, \\
\left(x_{1}, \ldots, x_{s}, u, x_{s+1}, \ldots, x_{j-1}, x_{j+1}, \ldots, x_{n}\right)^{\dagger}, & s \leq j-2 .\end{cases}
\end{aligned}
$$

Since

$$
0 \leq x_{1} \leq x_{1}^{*(j)}(u) \leq x_{2}^{*(j)}(u) \leq \cdots \leq x_{n}^{*(j)}(u) \leq x_{n},
$$

by Lemma 1, we see that $V_{n}\left(\mathbf{x}^{*(j)}(u), \alpha\right) \geq 0$. Furthermore, in view of the following simple fact,

$$
\left(\frac{x_{n-1}^{*(j) d_{n}}(u)+x_{n}^{*(j) d_{n}}(u)}{2}\right)^{\frac{1}{d_{n}}} \leq x_{n}^{*(j)}(u) \leq x_{n},
$$

we may see that

$$
\begin{aligned}
\sum_{j=1}^{n} \alpha_{j}-\frac{n(n-1)}{2} & =\sum_{j=1}^{n}\left[\alpha_{1}+\sum_{k=1}^{j-1} \Delta \alpha_{k}\right]-\frac{n(n-1)}{2} \\
& \geq \sum_{j=1}^{n}(j-1)-\frac{n(n-1)}{2}=0 .
\end{aligned}
$$


Thus, by (22), (23), (8) and (18), we have

$$
\begin{aligned}
& \left|V_{n}\left(\mathbf{x}^{(j)}(u), \alpha\right)\right|=V_{n}\left(\mathbf{x}^{*(j)}(u), \alpha\right) \\
& \leq\left(\prod_{j=1}^{n-1} j !\right)^{-1} v_{n}(\alpha) v_{n}\left(\mathbf{x}^{*(j)}(u)\right)\left(\frac{x_{n-1}^{*(j) d_{n}}(u)+x_{n}^{*(j) d_{n}}(u)}{2}\right)^{\frac{\sum_{j=1}^{n} \alpha_{j}-2^{-1} n(n-1)}{d_{n}}} \\
& \leq\left(\prod_{j=1}^{n-1} j !\right)^{-1} v_{n}(\alpha) v_{n}\left(\mathbf{x}^{*(j)}(u)\right) x_{n}^{\sum_{j=1}^{n} \alpha_{j}-2^{-1} n(n-1)} \\
& \leq v_{n}(\alpha)\left[1+\frac{2}{n(n-1)}\left(\sum_{i=0}^{\left[\frac{n-1}{2}\right]\left[\frac{n-1}{2}\right]} \sum_{j=1}^{1} \frac{1}{i+j}-\sum_{j=1}^{n-1} \frac{1}{j}\right)\right]^{\frac{n(n-1)}{2}} \\
& \times\left(\max _{1 \leq i \leq n-1} \Delta x_{i}\right)^{\frac{n(n-1)}{2}} x_{n}^{\sum_{j=1}^{n} \alpha_{j}-2^{-1} n(n-1)} \\
& =\left[1+\frac{2}{n(n-1)}\left(\sum_{i=0}^{\left[\frac{n-1}{2}\right]\left[\frac{n-1}{2}\right]} \frac{1}{i+j}-\sum_{j=1}^{n-1} \frac{1}{j}\right)\right]^{\frac{n(n-1)}{2}} \\
& \times v_{n}\left(\frac{\max _{1 \leq i \leq n-1} \Delta x_{i}}{x_{n}} \alpha\right) x_{n}^{\sum_{j=1}^{n} \alpha_{j}} \\
& =\left[1+\frac{2}{n(n-1)}\left(\sum_{i=0}^{\left[\frac{n-1}{2}\right]\left[\frac{n-1}{2}\right]} \frac{1}{i+j}-\sum_{j=1}^{n-1} \frac{1}{j}\right)\right]^{\frac{n(n-1)}{2}} \\
& \times v_{n}\left(\frac{\max _{1 \leq i \leq n-1} \Delta x_{i}}{x_{n}} \alpha\right) x_{n}^{\sum_{j=1}^{n} \alpha_{j}} \text {. }
\end{aligned}
$$

The proof is complete.

\section{Proof of Theorem 1}

First we point out that the generalized $\alpha$-polynomial in Theorem 1 satisfies

$$
\mathbf{f}(u)-\overline{\mathbf{y}}=\sum_{j=1}^{n} \frac{V_{n}\left(\mathbf{x}^{(j)}(u), \alpha\right)}{V_{n}(\mathbf{x}, \alpha)}\left(\mathbf{y}_{j}-\overline{\mathbf{y}}\right), u \in[0, \infty),
$$


for any $\overline{\mathbf{y}} \in R^{m}$. Indeed, the case where $n=2$ is easy. Suppose $n>3$. Since $\alpha \in \bar{\Omega}^{n}$, we see that

$$
\left|\begin{array}{ccccc}
1 & u^{\alpha_{1}} & u^{\alpha_{2}} & \ldots & u^{\alpha_{n}} \\
1 & x_{1}^{\alpha_{1}} & x_{1}^{\alpha_{2}} & \ldots & x_{1}^{\alpha_{n}} \\
1 & x_{2}^{\alpha_{1}} & x_{2}^{\alpha_{2}} & \cdots & x_{2}^{\alpha_{n}} \\
\vdots & \vdots & \vdots & \ddots & \vdots \\
1 & x_{n}^{\alpha_{1}} & x_{n}^{\alpha_{2}} & \cdots & x_{n}^{\alpha_{n}}
\end{array}\right|=0 .
$$

By Laplace expansion, we then have

$$
V_{n}(\mathbf{x}, \alpha)=\sum_{j=1}^{n} V_{n}\left(\mathbf{x}^{(j)}(u), \alpha\right) .
$$

Hence

$$
\begin{aligned}
\sum_{j=1}^{n} \frac{V_{n}\left(\mathbf{x}^{(j)}(u), \alpha\right)}{V_{n}(\mathbf{x}, \alpha)}\left(y_{j}-\overline{\mathbf{y}}\right) & =\sum_{j=1}^{n} \frac{V_{n}\left(\mathbf{x}^{(j)}(u), \alpha\right)}{V_{n}(\mathbf{x}, \alpha)} y_{j}-\frac{\sum_{j=1}^{n} V_{n}\left(\mathbf{x}^{(j)}(u), \alpha\right)}{V_{n}(\mathbf{x}, \alpha)} \bar{y} \\
& =f(u)-\bar{y} .
\end{aligned}
$$

We consider two cases:

$$
\theta=\min _{1 \leq i \leq n-1} \Delta \alpha_{i}=1 \quad \text { and } \quad \theta=\min _{1 \leq i \leq n-1} \Delta \alpha_{i} \neq 1 .
$$

In the former case, $\Delta \alpha_{j} \geq 1$ for $j=1,2, \ldots, n-1$. Therefore, by (24) and (21),

$$
\begin{aligned}
\|\mathbf{f}(u)-\overline{\mathbf{y}}\| & =\left\|\sum_{j=1}^{n} \frac{V_{n}\left(\mathbf{x}^{(j)}(u), \alpha\right)}{V_{n}(\mathbf{x}, \alpha)}\left(\mathbf{y}_{j}-\overline{\mathbf{y}}\right)\right\| \\
& \leq \sum_{j=1}^{n}\left\|\frac{V_{n}\left(\mathbf{x}^{(j)}(u), \alpha\right)}{V_{n}(\mathbf{x}, \alpha)}\left(\mathbf{y}_{j}-\overline{\mathbf{y}}\right)\right\| \\
& =\sum_{j=1}^{n} \frac{\left|V_{n}\left(\mathbf{x}^{(j)}(u), \alpha\right)\right|}{V_{n}(\mathbf{x}, \alpha)}\left\|\mathbf{y}_{j}-\overline{\mathbf{y}}\right\|
\end{aligned}
$$




$$
\begin{aligned}
& \leq \sum_{j=1}^{n} \frac{\rho_{n} v_{n}\left(\frac{\max _{1 \leq i \leq n-1} \Delta x_{i}}{x_{n}} \alpha\right) x_{n}^{\sum_{j=1}^{n} \alpha_{j}}}{V_{n}(\mathbf{x}, \alpha)}\left\|\mathbf{y}_{j}-\overline{\mathbf{y}}\right\| \\
& =\quad \rho_{n} \frac{v_{n}\left(\frac{\max _{1 \leq i \leq n-1} \Delta x_{i}}{x_{n}} \alpha\right) x_{n}^{\sum_{j=1}^{n} \alpha_{j}}}{V_{n}(\mathbf{x}, \alpha)} \sum_{j=1}^{n}\left\|\mathbf{y}_{j}-\overline{\mathbf{y}}\right\| \\
& =\quad \rho_{n} \frac{v_{n}\left(\frac{\max _{1 \leq i \leq n-1} \Delta x_{i}}{x_{n}} \alpha\right)}{V_{n}\left(\frac{\mathbf{x}}{x_{n}}, \alpha\right)} \sum_{j=1}^{n}\left\|\mathbf{y}_{j}-\overline{\mathbf{y}}\right\|,
\end{aligned}
$$

where

$$
\rho_{n}=\left[1+\frac{2}{n(n-1)}\left(\sum_{i=0}^{\left[\frac{n-1}{2}\right]\left[\frac{n-1}{2}\right]} \sum_{j=1}^{1} \frac{1}{i+j}-\sum_{j=1}^{n-1} \frac{1}{j}\right)\right]^{\frac{n(n-1)}{2}} .
$$

In the latter case, we have

$$
\min _{1 \leq i \leq n-1}\left(\frac{\alpha_{i+1}}{\theta}-\frac{\alpha_{i}}{\theta}\right)=1 .
$$

Let

$$
\mathbf{x}^{\theta}=\left(x_{1}^{\theta}, x_{2}^{\theta}, \ldots, x_{n}^{\theta}\right)^{\dagger}
$$

and

$$
\mathbf{x}^{\theta(j)}(u)=\left(x_{1}^{\theta}, x_{2}^{\theta}, \ldots, x_{j-1}^{\theta}, u^{\theta}, x_{j+1}^{\theta}, \ldots, x_{n}^{\theta}\right)^{\dagger}, j=1,2, \ldots, n .
$$

Then

$$
\begin{aligned}
\|\mathbf{f}(u)-\overline{\mathbf{y}}\| & =\left\|\sum_{j=1}^{n} \frac{V_{n}\left(\mathbf{x}^{\theta(j)}(u), \frac{\alpha}{\theta}\right)}{V_{n}\left(\mathbf{x}^{\theta}, \frac{\alpha}{\theta}\right)}\left(\mathbf{y}_{j}-\overline{\mathbf{y}}\right)\right\| \\
& \leq \rho_{n} \frac{v_{n}\left(\frac{\max _{1 \leq i \leq n-1}\left\{x_{i+1}^{\theta}-x_{i}^{\theta}\right\}}{x_{n}^{\theta}} \frac{\alpha}{\theta}\right)}{V_{n}\left(\frac{x^{\theta}}{x_{n}^{\theta}}, \frac{\alpha}{\theta}\right)} \sum_{j=1}^{n}\left\|\mathbf{y}_{j}-\overline{\mathbf{y}}\right\| \\
& =\rho_{n} \frac{v_{n}\left(\frac{\max _{1 \leq i \leq n-1}\left\{x_{i+1}^{\theta}-x_{i}^{\theta}\right\}}{\theta x_{n}^{\theta}} \alpha\right)}{V_{n}\left(\frac{\mathbf{x}}{x_{n}}, \alpha\right)} \sum_{j=1}^{n}\left\|\mathbf{y}_{j}-\overline{\mathbf{y}}\right\|,
\end{aligned}
$$


where

$$
\rho_{n}=\left[1+\frac{2}{n(n-1)}\left(\sum_{i=0}^{\left[\frac{n-1}{2}\right]} \sum_{j=1}^{\left[\frac{n-1}{2}\right]} \frac{1}{i+j}-\sum_{j=1}^{n-1} \frac{1}{j}\right)\right]^{\frac{n(n-1)}{2}}
$$

Our Theorem is thus proved.

As an immediate consequence of our Theorem and the inequality $\|\mathbf{f}(u)\| \leq$ $\|\overline{\mathbf{y}}\|+\|\mathbf{f}(u)-\overline{\mathbf{y}}\|$, we have the following

Corollary 1. Under the assumptions of Theorem 1, we have

$$
\begin{aligned}
\|\mathbf{f}(u)\| \leq & \|\overline{\mathbf{y}}\|+\left[1+\frac{2}{n(n-1)}\left(\sum_{i=0}^{\left[\frac{n-1}{2}\right]\left[\frac{n-1}{2}\right]} \sum_{j=1} \frac{1}{i+j}-\sum_{j=1}^{n-1} \frac{1}{j}\right)\right]^{\frac{n(n-1)}{2}} \\
& \times \frac{v_{n}\left(\frac{\max _{1 \leq i \leq n-1}\left\{x_{i+1}^{\theta}-x_{i}^{\theta}\right\}}{\theta x_{n}^{\theta}} \alpha\right)}{V_{n}\left(\frac{\mathbf{x}}{x_{n}}, \alpha\right)} \sum_{j=1}^{n}\left\|\mathbf{y}_{j}-\overline{\mathbf{y}}\right\| .
\end{aligned}
$$

We remark that Example 5.2 in [5, p. 1058] shows that the equality sign in (6) may hold.

\section{Remarks and Examples}

In Theorem 1 , the number $\theta=\min _{1 \leq i \leq n-1} \Delta \alpha_{i}$ can be an arbitrary positive number. However, in the case when the powers $\alpha_{1}, \alpha_{2}, \ldots, \alpha_{n}$ are nonnegative integers,

$$
\theta=\min \left\{\alpha_{j+1}-\alpha_{j} \mid j=1,2, \ldots, n-1\right\} \geq 1 .
$$

In particular, if there are two consecutive powers $\alpha_{i}$ and $\alpha_{i+1}$ such that $\alpha_{i+1}-\alpha_{i}=1$, then $\theta=1$. Such a choice can simplify matters since

$$
\frac{\max _{1 \leq i \leq n-1}\left\{x_{i+1}^{\theta}-x_{i}^{\theta}\right\}}{\theta x_{n}^{\theta}}=\frac{\max _{1 \leq i \leq n-1} \Delta x_{i}}{x_{n}} .
$$


In another direction, the number $\theta$ may be an arbitrary positive number but the sequence $\left\{x_{1}^{\theta}, x_{2}^{\theta}, \ldots, x_{n}^{\theta}\right\}$ in Theorem 1 of this paper may be uniform in the sense that

$$
x_{i+1}^{\theta}-x_{i}^{\theta}=h, i=1,2, \ldots, n-1,
$$

where $h$ is a constant.

In [5, p. 1047, Theorem 1.1], we obtained the following result: If $y \in \mathbf{C}^{n}$, $\mathbf{x} \in \Omega^{n}$ and $\alpha \in \bar{\Omega}^{n}$, then for $u \in\left[x_{1}, x_{n}\right]$, we have

$$
\|\mathbf{f}(u)\| \leq\left[\frac{2}{n(n-1)} \sum_{i=0}^{\left[\frac{n-1}{2}\right]\left[\sum_{j=1}^{\frac{n-1}{2}}\right]} \frac{1}{i+j}\right]^{\frac{n(n-1)}{2}} \frac{v_{n}\left(\frac{x_{n}^{\theta}-x_{1}^{\theta}}{\theta x_{n}^{\theta}} \alpha\right) \sum_{j=1}^{n}\left\|\mathbf{y}_{j}\right\|}{V_{n}\left(\frac{\mathbf{x}}{x_{n}}, \alpha\right)} .
$$

Now we prove the inequality (6) is strengthening of the inequality (26) in case $n=5$ or $n \geq 7$ and $x_{i+1}^{\theta}-x_{i}^{\theta}=h, i=1,2, \ldots, n-1$. To see this, take $\overline{\mathbf{y}}=0$ and

$$
(n-1)^{\frac{n(n-1)}{2}} v_{n}\left(\frac{\max _{1 \leq i \leq n-1}\left\{x_{i+1}^{\theta}-x_{i}^{\theta}\right\}}{\theta x_{n}^{\theta}} \alpha\right)=v_{n}\left(\frac{x_{n}^{\theta}-x_{1}^{\theta}}{\theta x_{n}^{\theta}} \alpha\right) .
$$

in (25), then

$$
\begin{aligned}
\|\mathbf{f}(u)\| \leq & {\left[1+\frac{2}{n(n-1)}\left(\sum_{i=0}^{\left[\frac{n-1}{2}\right]\left[\frac{n-1}{2}\right]} \sum_{j=1}^{\frac{1}{i+j}}-\sum_{j=1}^{n-1} \frac{1}{j}\right)\right]^{\frac{n(n-1)}{2}} } \\
& \times \frac{v_{n}\left(\frac{x_{n}^{\theta}-x_{1}^{\theta}}{\theta x_{n}^{\theta}} \alpha\right) \sum_{j=1}^{n}\left\|\mathbf{y}_{j}\right\|}{(n-1)^{\frac{n(n-1)}{2}} V_{n}\left(\frac{\mathbf{x}}{x_{n}}, \alpha\right)}
\end{aligned}
$$

Thus it suffices to show that when $n=5$ or $n \geq 7$,

$$
1+\frac{2}{n(n-1)}\left(\sum_{i=0}^{\left[\frac{n-1}{2}\right]} \sum_{j=1}^{\left[\frac{n-1}{2}\right]} \frac{1}{i+j}-\sum_{j=1}^{n} \frac{1}{j}\right)<\frac{2}{n} \sum_{i=0}^{\left[\frac{n-1}{2}\right]} \sum_{j=1}^{\left[\frac{n-1}{2}\right]} \frac{1}{i+j} .
$$

Indeed, note first (from [5, p. 1053, Lemma 2.8]) that

$$
\sum_{i=0}^{\left[\frac{n-1}{2}\right]} \sum_{j=1}^{\left[\frac{n-1}{2}\right]} \frac{1}{i+j}=\left(2\left[\frac{n-1}{2}\right]+1\right) \sum_{j=1}^{\left[\frac{n-1}{2}\right]} \frac{1}{\left[\frac{n-1}{2}\right]+j} .
$$


Therefore, it suffices to show (28) by showing

$$
\Phi_{n}:=\frac{2(n-2)}{n(n-1)}\left(2\left[\frac{n-1}{2}\right]+1\right) \sum_{j=1}^{\left[\frac{n-1}{2}\right]} \frac{1}{\left[\frac{n-1}{2}\right]+j}+\frac{2}{n(n-1)} \sum_{j=1}^{n-1} \frac{1}{j}>1
$$

when $n=5$ or $n \geq 7$. The cases $n=5,7,8,9$ can be proved by evaluating $\Phi_{5}, \Phi_{7}, \Phi_{8}, \Phi_{9}$ directly $\left(\Phi_{5} \simeq 1.0833, \Phi_{7} \simeq 1.4444, \Phi_{8} \simeq 1.0176, \Phi_{9} \simeq\right.$ 1.1859). As for the cases where $n \geq 10$, let us first note that the number

$$
m=\left[\frac{n-1}{2}\right] \geq \frac{n}{2}-1 .
$$

Then by the well known fact that $t>\ln (1+t)$ for $t \in(-1,+\infty) \backslash\{0\}$, we see that

$$
\begin{aligned}
\sum_{j=1}^{\left[\frac{n-1}{2}\right]} \frac{1}{\left[\frac{n-1}{2}\right]+j} & =\sum_{j=1}^{m} \frac{1}{m+j}>\sum_{j=1}^{m} \ln \left(1+\frac{1}{m+j}\right) \\
& =\ln \frac{2 m+1}{m+1}=\ln \left(2-\frac{1}{2 m+1}\right) \\
& \geq \ln \left[2-\frac{1}{2\left(\frac{n}{2}-1\right)+1}\right]=\ln \left(2-\frac{1}{n-1}\right) .
\end{aligned}
$$

Hence

$$
\begin{aligned}
\Phi_{n} & >\frac{2(n-2)}{n(n-1)}\left(2\left[\frac{n-1}{2}\right]+1\right) \sum_{j=1}^{\left[\frac{n-1}{2}\right]} \frac{1}{\left[\frac{n-1}{2}\right]+j} \\
& >\frac{2(n-2)}{n(n-1)}\left(2\left[\frac{n-1}{2}\right]+1\right) \ln \left(2-\frac{1}{n-1}\right) \\
& \geq \frac{2(n-2)}{n(n-1)}(n-1) \ln \left(2-\frac{1}{n-1}\right)=2\left(1-\frac{2}{n}\right) \ln \left(2-\frac{1}{n-1}\right) \\
& \geq 2\left(1-\frac{2}{10}\right) \ln \left(2-\frac{1}{10-1}\right)=1.01758>1
\end{aligned}
$$

as desired. 
We remark that (26) has been shown in [5] when $n \geq 2$ and the sequence $\left\{x_{1}^{\theta}, x_{2}^{\theta}, \ldots, x_{n}^{\theta}\right\}$ is not necessary uniform. However, the data points $\mathbf{y}_{1}$, $\mathbf{y}_{2}, \ldots, \mathbf{y}_{n}$ were assumed to be complex numbers only and that the reference vector $\overline{\mathbf{y}}$ is assumed to be $\mathbf{0}$.

While it is important to estimate the size of the interpolating $\alpha$-polynomial $\|\mathbf{f}(u)\|$ in reference to the origin, in applications it is also important to find upper bounds for $\|\mathbf{f}(u)-\overline{\mathbf{y}}\|$ where the reference point $\overline{\mathbf{y}}$ is an important landmark. For instance, given $n$ vectors $\mathbf{y}_{1}, \ldots, \mathbf{y}_{n}$ in $\mathbf{R}^{m}$, the Fermat point relative to these vectors is the vector $\overline{\mathbf{y}}$ such that

$$
\sum_{i=1}^{n}\left\|\mathbf{y}_{i}-\overline{\mathbf{y}}\right\|
$$

is minimized. In view of its definition, the Fermat point is clearly of practical importance. We will illustrate our result by an example in which the Fermat point is used as our reference vector.

Let $m=3$,

$$
\begin{aligned}
& p_{1}(u)=20[\ln (1+u)]^{2.1} \\
& p_{2}(u)=-20(2 \tan u-2 \sin u)^{1.1} \\
& p_{3}(u)=\left(e^{u}-1\right)^{5.4}
\end{aligned}
$$

and $p(u)=p_{1}(u)+p_{2}(u)+p_{3}(u)$, for $u \in[0,1]$. Let

$$
\mathbf{g}(u)=\left(u, p(u), 3 u^{2.1}\right)^{\dagger}, u \in[0,1] .
$$

Take 6 data pairs

$$
\begin{aligned}
&\left(x_{1}, \mathbf{g}\left(x_{1}\right)^{\dagger}\right)^{\dagger} \simeq(0,0,0,0)^{\dagger}, \\
&\left(x_{2}, \mathbf{g}\left(x_{2}\right)^{\dagger}\right)^{\dagger} \simeq(0.2,0.2,0.46124,0.10216)^{\dagger}, \\
&\left(x_{3}, \mathbf{g}\left(x_{3}\right)^{\dagger}\right)^{\dagger} \simeq(0.4,0.4,1.0338,0.43797)^{\dagger}, \\
&\left(x_{4}, \mathbf{g}\left(x_{4}\right)^{\dagger}\right)^{\dagger} \simeq(0.6,0.6,0.30170,1.02622)^{\dagger}, \\
&\left(x_{5}, \mathbf{g}\left(x_{5}\right)^{\dagger}\right)^{\dagger} \simeq(0.8,0.8,-2.36573,1.87763)^{\dagger}, \\
&\left(x_{6}, \mathbf{g}\left(x_{6}\right)^{\dagger}\right)^{\dagger} \simeq(1,1,-1.821068,3)^{\dagger} .
\end{aligned}
$$


Since

$$
\lim _{u \rightarrow 0^{+}} \frac{p_{1}(u)}{u^{2.1}}=20, \lim _{u \rightarrow 0^{+}} \frac{p_{2}(u)}{u^{3.3}}=-20, \lim _{u \rightarrow 0^{+}} \frac{p_{3}(u)}{u^{5.4}}=1,
$$

we will try to find the interpolating function in the form

$\mathbf{f}(u)=\left(\begin{array}{c}a_{1} \\ b_{1} \\ c_{1}\end{array}\right) u+\left(\begin{array}{c}a_{2} \\ b_{2} \\ c_{2}\end{array}\right) u^{2.1}+\left(\begin{array}{c}a_{3} \\ b_{3} \\ c_{3}\end{array}\right) u^{3.3}+\left(\begin{array}{c}a_{4} \\ b_{4} \\ c_{4}\end{array}\right) u^{4.3}+\left(\begin{array}{c}a_{5} \\ b_{5} \\ c^{5}\end{array}\right) u^{5.4}$,

where the exponent 4.3 is chosen so that $\theta=1$ (see the beginning remark in this section). By (5), we see that

$$
\mathbf{f}(u) \simeq\left(\begin{array}{c}
u \\
1.98663 u-3.59230 u^{2.1}+67.28831 u^{3.3}-164.27164 u^{4.3}+96.76793 u^{5.4} \\
3 u^{2.1}
\end{array}\right)
$$

for $t \in[0,1]$. See Figure 1 .

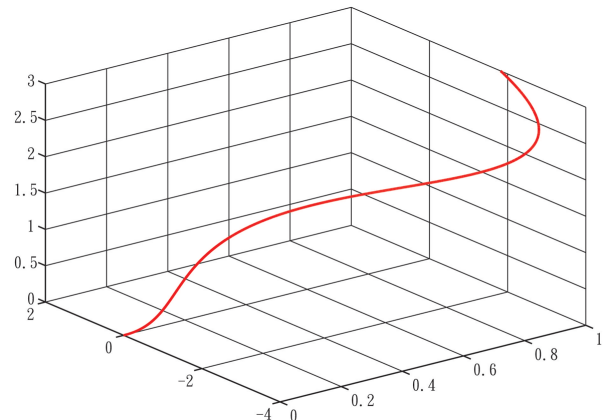

(a)

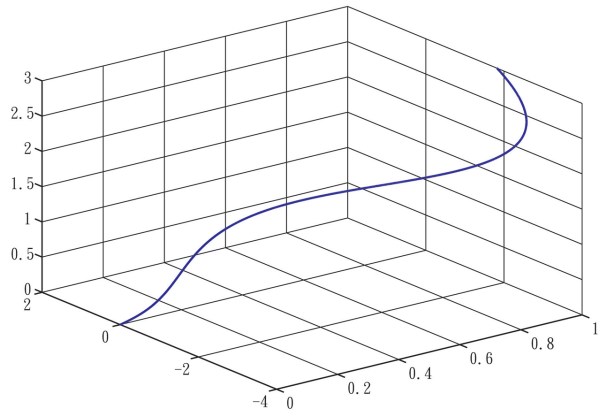

(b)

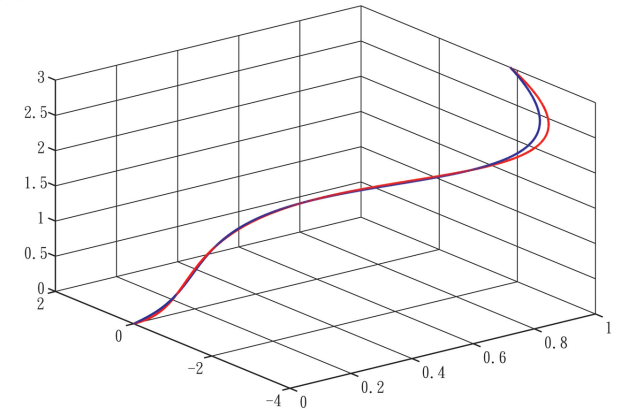

(c)

Figure 1 - Phase portraits of $\mathbf{g}$ in (a), $\mathbf{f}$ in (b), and $\mathbf{g}$ and $\mathbf{f}$ in (c). 
Next, the Fermat point $\overline{\mathbf{y}}=(p, q, r)^{\dagger}$ relative to the vectors $\mathbf{g}\left(x_{1}\right), \mathbf{g}\left(x_{2}\right)$, $\mathbf{g}\left(x_{3}\right), \mathbf{g}\left(x_{4}\right), \mathbf{g}\left(x_{5}\right)$ and $\mathbf{g}\left(x_{6}\right)$ is found by minimizing

$$
H(p, q, r)=\sum_{j=1}^{6}\left\{\left[\mathbf{g}\left(x_{j}\right)-(p, q, r)^{\dagger}\right]^{2}\right\}^{1 / 2}
$$

By using the computing tool Mathematica,

$$
\min H(p, q, r) \simeq H(0.48629,0.36004,0.55964) \simeq 7.81586,
$$

we see that

$$
(p, q, r)^{\dagger} \simeq(0.48629,0.36004,0.55964)^{\dagger} .
$$

Finally, the true bound is given by

$$
\max _{u \in[0,1]}\left\|\mathbf{g}(u)-(p, q, r)^{\dagger}\right\| \simeq 4.25302
$$

and

$$
\max _{u \in[0,1]}\left\|\mathbf{f}(u)-(p, q, r)^{\dagger}\right\| \simeq 4.06571,
$$

with $4.25302-4.06571=0.18731$.

The above example shows that Theorem 1 offers a closed ball with center $\overline{\mathbf{y}}$ that contains the approximating curve described by $\mathbf{f}$ over the interval $\left[x_{1}, x_{n}\right]$. By properly chosen $\overline{\mathbf{y}}$ as a reference point, tracking or control of spatial flying objects may then be feasible.

We close our investigation by remarking that results similar to Theorem 1 can be established if $\mathbf{R}^{m}$ is replaced by more general linear spaces endowed with appropriate algebraic operations and compatible norms, and many methods and techniques related to the mathematical inequalities used in this article can be found in [1-3, 5-9].

\section{REFERENCES}

[1] P.S. Bullen, D.S. Mitrinnovic and P.M. Vasic, Means and Their inequalities. Reidel, Dordrecht/Boston/Lancaster/Tokyo (1988).

[2] A.M. Fink and Z. Pales, What is Hadamard's inequality? Appl. Anal. Discrete Math., 1 (2007), 29-35. (Available at http://pefmath.etf.bg.ac.yu). 
[3] J.J. Wen and Z.H. Zhang, Vandermonde-type determinants and inequalities. AMEN, 6 (2006), 211-218.

[4] R. Aldrovandi, Special Matrices of Mathematical Physics: Stochastic, Circulant and Bell Matrices. World Scientific (2001).

[5] J.J. Wen and W.L. Wang, The inequalities involving generalized interpolation polynomial. Computer and Mathematics with Applications, 56 (2008), 1045-1058.

[6] J.E. Pecaric, J.J. Wen, W.L. Wang and L. Tao, A generalization of Maclaurin's inequalities and its applications. Mathematical Inequalities and Applications, 8(4) (2005), 583-598.

[7] J.J. Wen and Z.H. Zang. Jensen type inequalities involving homogeneous polynomials. Journal Inequalities and Applications. Volume 2010, Article ID 850215, 21 pages doi: 10.1155/2010/850215.

[8] J.J. Wen and W.L. Wang, The optimization for the inequalities of power means. J. Inequalities and Applications, Volume 2006, Article ID 46782, pages 1-25 doi: 10.1155/JIA/2006/46782.

[9] J.J. Wen and W.L. Wang, Chebyshev type inequalities involving permanents and their applications. Linear Algebra and its Applcations, 422(1) (2007), 295-303.

[10] P.J. Davies, Interpolation and Approximation. Dover (1975). 